\title{
SINGLE-WIRE HGMS OF COLLOIDAL PARTICLES: THE EVOLUTION OF CONCENTRATION PROFILES
}

A.J. Kramer, J.J.M. Janssen and J.A.A.J. Perenboom

High Field Magnet Laboratory and Research Institute for Materials

University of Nijmegen, Toernooiveld, NL-6525 ED Nijmegen, The Netherlands

Abstract Experimental Studies of HGMS in the colloidal regime of particle sizes frequently involve observations of the concentration profiles that are established around a magnetised filter-element. Attention is usually given to the density profiles in the regions of attractive particle-wire interaction, established after a considerable lapse of time. In this paper we will present and discuss the initial evolution on much shorter time-scales of the concentration profile of colloidal $\mathrm{Mn}_{2} \mathrm{O}_{3}$ particles around a magnetised steel wire, in the regions of repulsive interaction. Experimentally these observations are more convenient than the study of dynamic build-up in the attractive regions, because of the much shorter time-scales and correspondingly the relaxation of requirements on long-time colloid stability.

\section{Introduction}

HGMS (high-gradient magnetic separation) in the colloidal regime of particle sizes differs from conventional HGMS in the increased importance of diffusion effects, particle-wire-surface forces and interparticle interactions. A theoretical framework, in terms of particle concentration and particle flux rather than the behaviour of individual particles, has been formulated by Gerber and coworkers [1-3]. Experimental work in this field has involved studies of the capture efficiency for colloidal particles of wireand ball-filters [4,5], and observation of the concentration profiles that are established around a magnetised filter-element $[2,3,6]$ We will report some direct, microscopic observations of the evolution of the concentration profile of colloidal $\mathrm{Mn}_{2} \mathrm{O}_{3}$ particles around a magnetised steel wire. Unlike previous authors [2-6], we are mainly interested in observing the first stages of this evolution, and we see rapidly developing features that may provide a convenient testcase for the theoretical approach.

\section{Experimental set-up}

The intensity profile of light transmitted through the colloid surrounding the wire has been observed using a video-microscope Figure 1 shows the relative orientation of wire, magnetic field light path and cell windows in this experiment. The wire (AISI 430 , diameter $50 \mu \mathrm{m}$ ) was oriented parallel to the gravitational force, to avoid field-induced convection [6] that was observed to greatly disturb experiments with the wire oriented horizontally The cell, which has a volume of $20 \times 7 \times 1 \mathrm{~mm}^{3}$, was illuminated from the backside with diffuse white light. The video-microscope

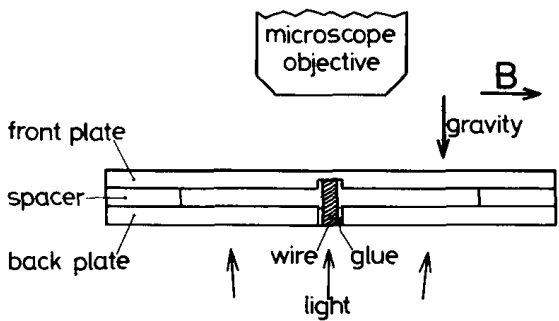

Figure 1: The geometry of the experiment: side view consists of two microscope lenses, which cast an image of the wire and its surroundings onto the sensitive element of a CCD-camera. A monitor and videorecorder were connected to the camera to observe and store the images. Some video images were digitised and the data were processed in order to extract the concentration profiles.

The colloid was prepared using commercially available $\mathrm{Mn}_{2} \mathrm{O}_{3}$ powder that had been subjected to additional ball milling. The ball-milled powder was dispersed in a $2.0 \mathrm{~g} / 1$ solution of $\mathrm{Na}_{4} \mathrm{P}_{2} \mathrm{O}_{7}$. $10 \mathrm{H}_{2} \mathrm{O}$. This colloid was filtered through $0.4 \mu \mathrm{m}$ membrane filters. Just before use in an experiment the colloid was ultrasonically agitated, although it appeared to be stable even in a magnetic field on the time-scales of interest.
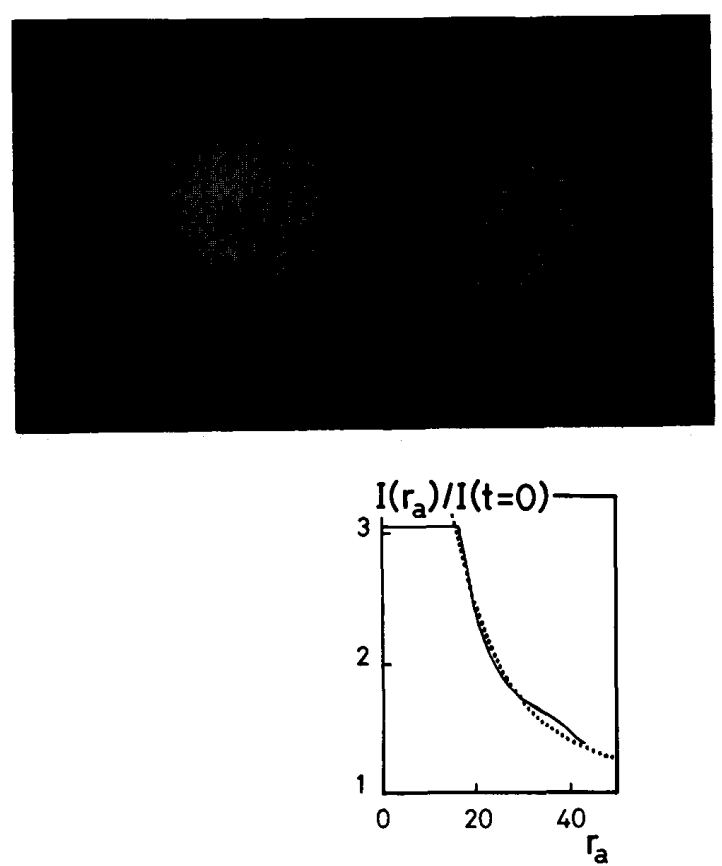

Figure 2: a) Typical example of the recorded image of a developing profile of transmitted light through a $\mathrm{Mn}_{2} \mathrm{O}_{3}$ colloid (taken 30 seconds after switching on a field of $1.3 \mathrm{~T}$ ); the maximum repulsive interaction $\left(\theta=90^{\circ}\right)$ is along the horizontal axis in this picture. b) Long-time intensity profile recorded along $\theta=90^{\circ}$ (solid line). Dotted line: fit to the analytical equilibrium solution (Eq. 6). The cut-off just above $I\left(r_{a}\right) / I(0)=3$ is due to camera overload.

\section{Results}

In Fig. $2 \mathrm{a}$ a typical example of the profile is shown that will develop when a magnetic field is applied. The most remarkable feature observed is that a rather sharp boundary moves outward in the regions where the magnetic particle-wire interaction is repulsive. The experiment was repeated for several different magnetic fieldstrengths between 0.3 and $1.3 \mathrm{~T}$ 


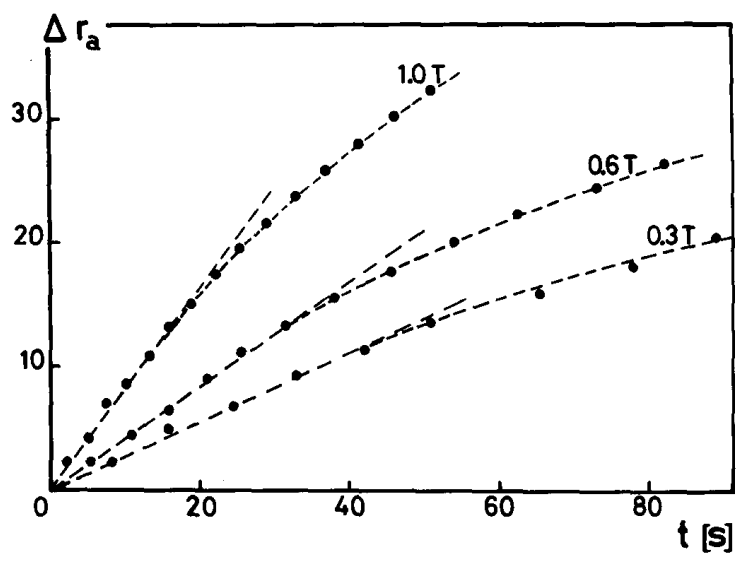

Figure 3: Position of the moving boundary in the region of repulsive interaction (i.e. along the $\theta=90^{\circ}$ direction) relative to a mark close to $r_{a}=10$ as a function of time, for some representative fieldstrengths.

The time in which this boundary would move beyond the screen-edge (about 40 wire radii from the wire) was typically about one minute. On the monitor, observed with the eye, the boundary is quite pronounced. The digitised intensity profiles also show a step in intensity at the boundary position, but it is less sharp than expected from the direct observation. It is possible that optical illusion or monitor artifacts suggest a sharp boundary to the eye. It is important to note that the intensity profile observed in the region of repulsive interaction will hardly change in time after the boundary has moved beyond the screen-edge. This fact suggests that an equilibrium is established between the particle flux due to the repulsive magnetic particle-wire interaction and the diffusive flux caused by the concentration gradient. Figure $2 \mathrm{~b}$ shows an example of such a nearly-equilibrium intensity profile for $\theta=90^{\circ}$, where a cylindrical coordinate system is used with the wire along the $z$-axis and the direction of the applied field defining $\theta=0^{\circ}$.

At the initial stages of the process, it is difficult to make reliable and reproducible observations; this is because the motion of the boundary is quite fast, and also it takes a few seconds for the magnetic fieldstrength to reach its final value. In order to obtain quantitative data on the motion of the boundary at fixed magnetic fieldstrengths, we have mounted a mm-scale on the monitor along the $\theta=90^{\circ}$ direction. For each different fieldstrength the moment in time at which the boundary reached a mark close to $r_{a}=10$ was chosen as $t=0$ (note that $r_{a}=r / a$, where $a$ is the radius of the magnetised wire). Figure 3 shows the position of the

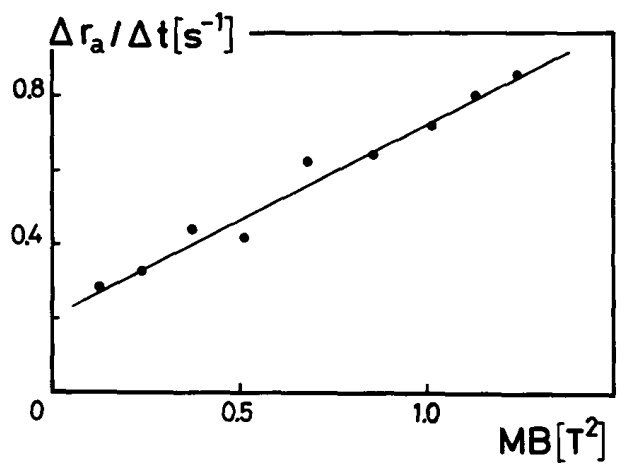

Figure 4: Propagation velocity of the boundary just after passing the mark near $r_{a}=10$ (cf. the initial slope in Fig. 3) as a function of $M B$, the product of the wire magnetisation and the applied magnetic field. boundary as it develops relative to this mark as a function of time, for some representative fieldstrengths. The moment that the boundary passed a certain value $r_{a}$ was measured several times by replaying the event, and we obtained an accuracy better than the size of the symbols in the graph. The curves are nearly straight initially: this indicates that the boundary moves with an almost constant velocity in the range $r_{a} \simeq 10-25$. The velocity is shown as a function of the product $M B$ of the magnetisation of the wire and the applied field in Fig. 4.

\section{Discussion}

Although, as we have already mentioned, the establishment of an equilibrium situation indicates that diffusion effects are significant in this case, it is instructive to show more explicitly that our observations are incompatible with a purely mechanical model. In such a model, the boundary would be identified with the layer of particles present at the wire surface when the field is applied, and the gradual change in the transmission of light at larger distances would be attributed to the colloid's polydispersity. Consider a particle of radius $b$ and volume susceptibility $\chi$ that moves in a fluid of viscosity $\eta$ near a magnetised wire. Along the $\theta=90^{\circ}$ axis the balance between the magnetic particle-wire interaction and the Stokes drag leads to:

$$
\frac{\mathrm{d} r_{a}}{\mathrm{~d} t^{\prime}}=r_{a}^{-3}-K r_{a}^{-5}
$$

where $t^{\prime}=2 \chi M B b^{2} t / 9 \mu_{0} \eta a^{2}$ is a dimensionless time, and $K=$ $M / 2 B \leq 1$. If we fix the integration constant with the condition that the particle is at $r_{a}^{*}$ at time $t^{*}$, the solution of Eq. (1) is:

$$
t^{\prime}-t^{*}=\frac{1}{4}\left(r_{a}^{4}-r_{a}^{* 4}\right)+\frac{1}{2} K\left(r_{a}^{2}-r_{a}^{* 2}\right)+\frac{K^{2}}{2} \ln \frac{r_{a}^{2}-K}{r_{a}^{* 2}-K} .
$$

According to this equation a particle that starts at $r_{a}^{*}=1$ needs an amount of time $t^{\prime} \approx 6.4 \times 10^{5}$ to reach the screen-edge at $r_{a} \approx 40$. Using $\chi=5.3 \times 10^{-3}$ for $\mathrm{Mn}_{2} \mathrm{O}_{3}, a=25 \mu \mathrm{m}, \eta=10^{-3} \mathrm{~kg} / \mathrm{ms}$ for water, and $b \leq 0.2 \mu \mathrm{m}$ (because the colloid passed through a 0.4 $\mu \mathrm{m}$-pore membrane filter), we find $t>9600 \mathrm{~s}$ for $M B=1.1 \mathrm{~T}^{2}$, which is orders of magnitude larger than the experimentally observed time-scale of about one minute. Clusters with a radius in the $2.5 \mu \mathrm{m}$ range would be necessary to obtain some agreement of Eq. (2) with the experiments. The presence or formation of such large clusters is very unlikely for more than one reason: because of the pre-treatment of the colloid (filtering through a $0.4 \mu \mathrm{m}$ filter, and ultrasonic agitation just before the experiment) and because of the stability observed against sedimentation and magnetic flocculation (in the fieldstrengths used in this experiment)

A statistical description of the experiment would start with a diffusion equation [1], which can be cast in a dimensionless form:

$$
\frac{\partial n}{\partial \tau}=\nabla_{a} \cdot\left(\nabla_{a} n+n \nabla_{a} V / k_{\mathrm{B}} T\right)
$$

Here $n$ is the normalised particle number density ( $n=1$, initially), $\tau=k_{\mathrm{B}} T t / 6 \pi \eta b a^{2}, V$ is the particle-wire interaction energy, and the subscript " $a$ " does indicate that the spatial derivatives should be taken with respect to the dimensionless position $r_{a}$. Here we will assume that in $V$ only the magnetic interaction needs to be considered:

$$
V / k_{\mathrm{B}} T=-W\left(r_{a}^{-4} K / 2+r_{a}^{-2} \cos 2 \theta\right),
$$

where $W=2 \pi b^{3} \chi M B / 3 \mu_{0} k_{\mathrm{B}} T$. When we choose the boundary condition that the flux through the wire surface should be zero (which means assuming an impenetrable, non-sticking surface), the equilibrium solution reads:

$$
n=\exp \left(-V / k_{\mathrm{B}} T\right) .
$$


If one assumes a Lambert-Beer relation between the intensity of the transmitted light and the particle number density, the transmission of light after establishment of an equilibrium will be given by:

$$
I=I_{0} \exp \left(-C \exp \left(-V / k_{\mathrm{B}} T\right)\right),
$$

where $C$ is a parameter that depends on the extinction coefficient of the particles, their initial number density and the length of the lightpath through the colloid. $I_{0}$ is the incident intensity. A two-parameter fit of the nearly-equilibrium profile of Fig. $2 \mathrm{~b}$ using Eq. (6) gives an estimate of about 320 for $W$. By substituting the appropiate values for the other parameters in the expression for $W$, we deduce a particle radius $b \approx 50 \mathrm{~nm}$, which is acceptable on the basis of the pre-treatment and the stability of the colloid. This does not mean however that this value should also be valid for the particles that are involved in the moving boundary. In a polydisperse colloid it may well be that the largest particles present lead to the phenomenon of the moving boundary, while smaller ones remain in the vicinity of the wire and establish an equilibrium situation as described by Eq. (5). In the absence of flocculation, the largest value of $W$ possible in our experiment would be about 20000 , for the maximum particle size $b=0.2 \mu \mathrm{m}$ and $M B \simeq 1.3 \mathrm{~T}^{2}$.

In order to obtain a more detailed comparison between theory and experiment we have started a numerical analysis of the time-dependent diffusion equation. Initially we have studied the $\theta=90^{\circ}$ version of Gerbers 1D model [1]. In Fig. 5 some timedependent concentration profiles are shown, calculated using an implicit finite-differencing approach $[7,8]$. An important question is which point on such a profile should be identified with the moving boundary in our experiments? At present the most plausible choice seems to be the maximum in $n\left(r_{a}\right)$, which corresponds to a minimum in the transmitted intensity. Even if this maximum does not coincide with the experimentally observed boundary, its propagation will give a measure of the time-scale within which the largest change in the corresponding density profile will take place: It was observed in the experiments that the intensity profile does not change much after the boundary has passed the screen-edge, and indeed the calculated density profiles are very similar to the equilibrium profile that can be calculated using Eq. (5) (cf. Fig. 5) when the maximum in $n\left(r_{a}\right)$ has moved to larger distances. From the definitions of $t^{\prime}, W$ and $\tau$ we obtain $t^{\prime}=2 W \tau$. Using this relation we can compare the propagation of the maximum in $n\left(r_{a}\right)$ with calculations from Eq. (2). We find that the maximum indeed moves faster than an individual particle would move for the same experimental parameters. The ratio depends on $W$ and on the specific value of $r_{a}$ under consideration. For $W$ between 25 and 450 , and $r_{a}$ up to 40 , the ratio is found to vary between about 1.5 and 5 . So, the 1D diffusion model appears to be an improvement relative to a purely mechanical model, but the time-scale predicted is still too long to agree with our experimental observations.

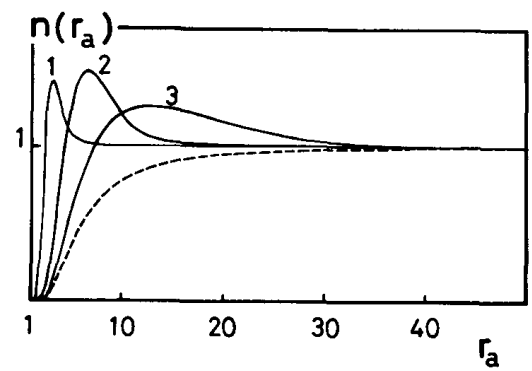

Figure 5: Evolution of the concentration profile in the $\theta=90^{\circ}$ version of Gerber's $1 \mathrm{D}$ diffusion model for $W=25$ and $K=0.6$. The curves 1 to 3 correspond to $\tau=0.2,5$ and 50 respectively. The dashed line represents the equilibrium profile calculated using Eq. (5).
Our preliminary calculations for a $2 \mathrm{D}$ model suggest that this will give shorter times, as can be expected from physical arguments: in a $1 \mathrm{D}$ model only radial particle transport is possible, while a 2D model also allows azimuthal transport. The transition from the inital situation to the equilibrium distribution (which are both the same for $\theta=90^{\circ}$ in 1D or 2D) will thus occur faster. Unfortunately finite-differencing techniques turned out to be very time-consuming in the $2 \mathrm{D}$ case, especially if one wishes to model the propagation of the maximum to larger distances. At present we are considering an alternative approach where we first make the substitution $n(r, \theta, \tau)=f(r, \theta, \tau) \exp \left(-V(r, \theta) / k_{\mathrm{B}} T\right)$ in Eq. (3). As a second step, the 2D equation for $f$ is replaced by an equivalent series of coupled 1D equations, by expressing the angle-dependence of $f$ as a Fourier series. Since the equilibrium solution for $f$ is simply unity (see Eq. (5)), higher order terms are expected to decay rather rapidly when the density profile evolves towards equilibrium, and only a few terms have to be maintained at large values of $\tau$. A similar approach has already been succesfully used in a recent paper [9] to solve the $2 \mathrm{D}$ diffusion equation that governs magnetic flocculation.

\section{Conclusions}

When an external magnetic field is applied to a colloid that surrounds a magnetisable filter-element, the initially homogeneous density will evolve towards a new equilibrium distribution (which may include some static particle capture). In the region of repulsive magnetic particle-wire interaction this evolution leads to the distinct phenomenon of a rather sharp boundary that moves outward to larger distances. Observation of the shape and propagation velocity of this boundary provides means to check and refine the theoretical models. Experimentally these observations are more convenient than a study of dynamic build-up in the attractive regions, because of the much shorter time-scales and correspondingly the relaxation of requirements on long-time colloid stability.

Acknowledgements We would like to thank A.F. van Etteger for making the CCD camera and the lenses available to us, and S.J.M. Oosterbaan for his help with the digitising of some of the images. Stimulating discussions with A.P. van Gelder are also gratefully acknowledged. Part of this work was supported by the Stichting voor Fundamenteel Onderzoek der Materie (FOM) and the Stichting voor de Technische Wetenschappen (STW), with financial support from the Nederlandse Organisatie voor Wetenschappelijk Onderzoek (NWO).

\section{References}

1] R. Gerber, IEEE Trans. Magn. MAG-20, 1159 (1984).

[2] M. Takayasu, R. Gerber and F.J. Friedlaender, IEEE Trans. Magn. MAG-19, 2112 (1983).

[3] R. Gerber, M. Takayasu and F.J. Friedlaender, IEEE Trans. Magn. MAG-19, 2115 (1983)

[4] H.Th.J. Reijers, R.P.A.R van Kleef, H.W. Myron, P. Wyder and M.R. Parker, J. Magn. Magn. Mater. 58, 123 (1986).

[5] M.F. Haque, R. Aidun, C. Moyer and S. Arajs, J. Appl. Phys 63, 3239 (1988).

6] E. Blums, A.Y. Chukhrov and A. Rhimsa, Proc. $4^{\text {th }}$ World Filtration Congress; Ostend, Belgium, 22-25 April 1986, p. $3.37-3.43$.

[7] W.F. Ames, Numerical Methods for Partial Differential Equations (Academic Press, New York, 1977).

[8] W.H. Press, B.P. Flannery, S.A. Teukolsky and W.T. Vetterling, Numerical Recipes in $C$ (Cambridge University Press, New York, 1988).

9] J.J.M. Janssen, J.J.M. Baltussen, A.P. van Gelder and J.A.A.J. Perenboom, submitted to J. Phys D: Appl. Phys. 